1 When citing this article: Van den Bulcke, J., De Windt, I., Defoirdt, N., De Smet, J., Van Acker, J. (2011).

2 Moisture dynamics and fungal susceptibility of plywood. International Biodeterioration and 3 Biodegradation 65(5): 708-716.

$4 \quad$ http://dx.doi.org/10.1016/j.ibiod.2010.12.015 


\title{
5 Moisture dynamics and fungal susceptibility of plywood
}

6

\author{
Van den Bulcke Jan ${ }^{1 *}$, De Windt Imke ${ }^{1}$, Defoirdt Nele ${ }^{1}$, De Smet Jordi ${ }^{1}$, Van Acker Joris ${ }^{1}$
}

${ }^{1}$ Laboratory of Wood Technology, Department of Forest and Water Management, Faculty of Bioscience Engineering, Ghent University, Ghent

* corresponding author: Jan.VandenBulcke@UGent.be

Laboratory of Wood Technology

Department of Forest and Water Management, Faculty of Bioscience Engineering

Coupure Links 653, 9000 Ghent, Belgium

Tel: +32(0)9 2646124

Fax: +32(0)9 2646233

\section{Abstract}

Engineered wood products are widely used in construction and transportation. Plywood has one of the best physical and mechanical properties for application under moist conditions in use class 3, i.e. exterior exposure without ground contact. Yet a profound knowledge on their moisture dynamics and fungal susceptibility is a prerequisite for proper use. In this paper the results of more than two years of continuous recording of the moisture content of different plywood types in exterior exposure is presented. Clear differences concerning moisture dynamics are apparent regarding wood species, glue type and veneer thickness. X-ray tomography scans literally offer insight in the weathering state of the veneer layers and glue lines. Fungal susceptibility of the samples relates to wood species and glue type mainly. Furthermore, edge sealing for fungal testing is necessary to represent in service situations and gives distinct differences with non-sealed test results. Seemingly, there is a relationship between the accumulated moisture content and layered structure of the plywood, but an unequivocal link with fungal decay is not straightforward.

Keywords: plywood; continuous moisture measurement; fungal susceptibility; X-ray tomography; doseresponse 


\section{7}

38

39

40

41

42

43

44

45

46

47

48

49

50

51

52

53

54

55

56

57

58

59

60

61

62

63

64

65

66

67

68

69

70

71

72

73

74

75

76

77

78

\section{Introduction}

Officially, more than three billion $\mathrm{m}^{3}$ of wood is harvested yearly from forests all over the world (FAO 2009). The depletion of durable wood (Tascioglu and Tsunoda 2010) and the shortage of quality timber (Chung et al. 1999) necessitate the use of less durable and lesser known wood species as well as the use of engineered wood products. Furthermore, efforts to shift to more low-energy, environmental friendly building materials and the endeavour at minimizing costs (Krüger and Laroca 2010) have also created the search for adopted or new building materials and building concepts with an important role for these engineered products. The advantage of the latter is that they can be manufactured with a wide range of properties for a wide range of purposes. Among the engineered wood products, plywood is the wood based panel showing one of the best physical and mechanical properties for application in use class 3 , i.e. exterior exposure without ground contact. In 2008 the production and consumption in the EU-27 were respectively 3.16 million $\mathrm{m}^{3}$ and 6.31 million $\mathrm{m}^{3}$ (FEIC 2009), stressing its importance on an international scale. The mechanical and physical criteria to be met for construction purposes in general and more specifically for outdoor application are well defined. Appropriate physico-mechanical durability for exterior applications in transportation or construction systems can be guaranteed by assessment of the glue bond quality and mechanical characteristics. Where it concerns the requirements for biological durability needed when using plywood in outdoor applications, the current European standardization remains vague and insufficient. According to EN 636 (2003) the durability should be addressed as outlined in EN 335-3 (1995) while guidance on factors affecting durability and on precautionary measures which may be considered as necessary can be found in ENV 1099 (1998). But the latter offers only very brief and incomplete information (De Smet and Van Acker 2006). However, since several plywood types based on less durable wood species show good performance in exterior conditions, more accurate knowledge on the biological durability is needed in order to be able to classify them according to durability performance. In this regard the study of their moisture dynamics is crucial, all the more because it is generally known that moisture has a significant influence on the mechanical and physical properties of wood, plywood and other wood based materials (Dinwoodie 2000). Practical experience with plywood shows that it outperforms solid wood homologues and underpins a separate approach. In Van den Bulcke et al. (2009b) a new set-up was introduced to monitor the moisture content (MC) of plywood continuously on a weighted mass basis (CMM = Continuous Moisture Measurement). In the present paper the results of more than two years of CMM testing of a selection of plywood types are processed and presented. X-ray radiography and tomography scans are taken for visual aid at the interpretation of moisture measurements. Furthermore, comparison is made with fungal testing in the laboratory in order to relate the outdoor moisture behaviour with fungal decay susceptibility. Several research questions are highlighted, without the intention to rank different plywood types according to performance: How do different plywood types behave in terms of moisture dynamics during two years of outdoor weathering? Are differences discernable regarding glue type, wood species and veneer thickness? Are effects of weathering visible with X-ray radiography and tomography? What is the fungal susceptibility of plywood? Do measurements of plywood $M C$ and the combined dose of $M C$ and temperature determined in the CMM set-up relate to fungal lab testing?

\section{Material and Methods}




\subsection{Test material}

The research presented in this paper is part of the work performed in a European Research Project called PLYBIOTEST. In this project a total of 60 different kinds of plywood produced by European plywood companies were tested for the biological performance in exterior out of ground application. For the purpose of this paper a selection is made of 10 plywood types which can be regarded as typical plywood available on the European market (Table 1). Both tropical hardwood species such as okoumé (Aucoumea klaineana) and sapelli (Entandrophragma cylindricum) as well as hard- and softwood species such as birch (Betula spp), poplar (Populus spp), spruce (Picea abies) and maritime pine (Pinus pinaster) are used. From this selection, the birch (b1) and the poplar plywood glued with UMF (p1) and PF (p3) have a coated equivalent (phenolic film) which is added for comparison.

\subsection{CMM set-up}

For detailed information on the CMM set-up and accompanying sample preparation, the reader is referred to Van Acker and De Smet (2007) and Van den Bulcke et al. (2009b). Briefly, the CMM test set up consists of a wooden table upon which two parallel series of single load cells are fixed. The load cells are mounted onto small inert plates adjustable with screws in the four corners for levelling. On top of the load cells aluminium T-shaped holders are fastened. Plywood specimens measuring $150 \mathrm{~mm} \times 150$ $\mathrm{mm}$ with sealed edges are mounted on these holders. All load cells are calibrated such that the recorded voltages can be converted to weight values. $M C$ of the test specimens was determined after the experiment by oven drying the plywood samples and used as a reference for converting weight signals to actual MC. Adjacent to the CMM set up a fully equipped weather station is installed consisting of a solar radiation sensor, a tipping bucket rain gauge, a relative humidity probe, a thermometer, an anemometer and a wind vane. All weather and moisture data are collected with an interval of five minutes.

\subsection{X-ray radiography and tomography}

The X-ray equipment of the Centre for X-ray Tomography (www.ugct.ugent.be) is used for evaluation of the plywood samples after weathering. A more detailed description of the tomographical set-up is found in Van den Bulcke et al. (2009a). The weathered samples are mounted close to the detector and X-ray radiographies are taken for one sample for each plywood species. A resolution of approximately $110 \mu \mathrm{m}$ is obtained. For the detailed 3D scans, small subsamples are taken in order to visualize the different veneer layers and the glue surfaces. The edge length of the cubic subsamples is equal to the thickness of the plywood board. Eight cone beam scans were taken sequentially. The sample is rotated for $360^{\circ}$ and a projection image is taken every $0.45^{\circ}$. All these projection images are reconstructed and an approximate voxel size of 40 micron is obtained. Sample preparation, scanning and reconstruction took approximately 30 minutes. Volumes are visualized with VGStudio MAX®.

\subsection{Fungal susceptibility}

To evaluate the standard durability test methodology, an ENV 12038 (1999) test was set up for a selection of plywood types. Obligatory test fungi as mentioned in the ENV 12038 are Coniophora puteana (Schumacher ex Fries) Karsten (strain BAM Ebw. 15) and Pleurotus ostreatus (Jacquin ex Fries) 
Quélet (strain FPRL 40C). Specimen sizes are $50 \times 50 \mathrm{~mm}$ and six replicates per fungus are used. A selection of eight plywood types was edge sealed on four sides with two layers of a two-component polyurethane finish to test the effect of sealing on decay. Every specimen is conditioned at $20^{\circ} \mathrm{C} \pm 0.5^{\circ} \mathrm{C}$ and $65 \% \pm 1 \% \mathrm{RH}$ until constant mass is reached, weighed, sterilized and placed in a culture vessel on agar overgrown with the test fungus. The incubation period lasts for 16 weeks. At the end of incubation the test specimens were withdrawn from the culture vessels. The adhering mycelium was carefully removed and the specimens were weighed to the nearest $0.01 \mathrm{~g}$ to determine the wet mass enabling to calculate the moisture content at the end of the test. The test specimens were placed in the oven at $103^{\circ} \mathrm{C} \pm 2^{\circ} \mathrm{C}$ until the specimens reached constant mass and were weighed to determine the dry mass. The mass loss of each specimen was calculated by expressing the mass loss as a percentage of the final dry mass. A test product is designated as fully resistant to attack by wood-rotting Basidiomyceta when (a) the mean loss in mass of the test specimens is less than $3 \%(\mathrm{~m} / \mathrm{m})$, and $(\mathrm{b})$ not more than one test specimen has suffered a loss in mass greater than $3 \%$ but less than $5 \%$. As formaldehyde emission and other volatile components can have an inhibitory effect on the test results, test specimens are subjected to a preconditioning of 12 weeks in a well ventilated room at $20^{\circ} \mathrm{C} \pm 0.5^{\circ} \mathrm{C}$ and $65 \% \pm 1 \%$ RH before testing.

\subsection{Analysis of the $\mathrm{CMM}$ data and the Brischke model}

All data were converted to hourly data by averaging and missing data were excluded from analysis. For all plywood specimens, MC histograms were calculated. Further, Time of Wetness (ToW) was also determined based on the number of days above the $20 \%$ and $25 \%$ moisture limits which are used as guiding values for comparison of the different plywood types. Furthermore, the total dose that each plywood type received based on the $\mathrm{MC}(\%)$ of the plywood samples and the temperature $\left(\mathrm{T}^{\circ} \mathrm{C}\right)$ measured by the weather station (material temperature was not known) was calculated using following formula (Brischke and Rapp 2008):

$d=d_{M C} \times d_{T}$

with $d_{M C}$ the $M C$ induced daily dose and $d_{T}$ the temperature induced daily dose:

$\mathrm{d}_{M C}=6.75 \times 10^{-10} \mathrm{MC}^{5}-3.50 \times 10^{-7} \mathrm{MC}^{4}+7.18 \times 10^{-5} \mathrm{MC}^{3}-7.22 \times 10^{-3} \mathrm{MC}^{2}+0.34 \mathrm{MC}-4.98$

if $M C \geq 25 \%$

$d_{T}=1.8 \times 10^{-6} \mathrm{~T}^{4}+9.57 \times 10^{-5} \mathrm{~T}^{3}-1.55 \times 10^{-3} \mathrm{~T}^{2}+4.17 \times 10^{-2} \mathrm{~T}$

Clearly, as air temperature is measured and not material temperature, the $d_{T}$ values are the same for all plywood samples but due to the multiplicative nature of total dose calculation, this can have an important influence. Although the dose-response approach of Brischke and Rapp (2008) is based on the relation between in-service fungal growth, temperature and the available moisture, it is used here as a meaningful ranking value for the different plywood types.

\section{Results}




\subsection{X-ray radiography}

A general view on the different plywood types used is given in Figure 1 by means of X-ray radiography. Obviously, as intensity is an integration along the thickness of the material, darker zones represent higher attenuating regions caused by a higher density of the wood species, amount and thickness of glue lines and veneer layers. Therefore the thick b2 species and the dense sap species are in general darker than for instance the p3 sample, although its total thickness is larger than sap. A striking example is the $\mathrm{mp}$ panel, showing both darker and lighter streaks due to large early- and latewood bands. Larger defects throughout the sample are visible as well, such as knots and cracks. The two lighter points on each panel are the screw holes for attachment at the T-shaped holders. The darker halo around each hole is the remainder of a silicone sealant that was used to prevent water penetration along the screw thread.

\subsection{Analysis of the CMM data and X-ray tomography}

The CMM set up started running in June 2006. This study contains data of more than two years of exposure. Weather details as recorded during this period are presented in Table 2. Solar radiation is calculated over 24 hours. Figure 2 gives a graphical overview of the daily MC pattern for maritime pine together with daily climate data (rainfall, relative humidity $(\mathrm{RH})$ and temperature). The combined influence of rain and temperature is distinguishable when looking at the $\mathrm{MC}$ profile. Continuous raining induces moisture accumulation, causing high MC during colder periods when temperature and solar irradiation are too low to force drying of the samples. The sharp peaks at the end of the measuring periods are believed to be caused by cracks in the top veneer and the glue line beneath it. It should be remarked that these measurements are an average of the sample and do not give a decisive answer about the moisture distribution in the sample. Although the relation between rain events and absorption / desorption of moisture by the plywood samples can be seen in this kind of graphs, for long time series analysis it is useful to assess them differently. Straightforward analysis of the moisture dynamics is histogram plotting, displaying the amount of days a sample had a specific MC as shown in Figure 3. The two lines represent the critical $20 \%$ and $25 \%$ moisture content limits. They provide a means to visually assess a plywood sample. Another feature observed in some plywood species, is a differentiation between high and low absorbing samples. High absorbing samples were analyzed separately (black bar graphs in Figure 3 ) and not averaged in the low absorbing samples. Clearly, most of the plywood species exceed the $20 \%$ and $25 \% \mathrm{MC}$ limit considerably. The effect of the thickness difference between $15 \mathrm{~mm}$ and $18 \mathrm{~mm}$ UMF glued poplar plywood ( $\mathrm{p} 1$ and $\mathrm{p} 2$ ) is small, although the distribution for $\mathrm{p} 1$ is smaller and sharper, most probably due to a thinner top veneer. Glue-type is a more decisive parameter for the moisture behaviour of plywood. PF-glued $18 \mathrm{~mm}$ thick poplar plywood (p3) is reaching higher MCs during several days than the UMF-glued variant ( $\mathrm{p2}$ ). The spruce (sp) and maritime pine ( $\mathrm{mp}$ ) plywood also exhibit a high absorption. Okoumé and especially sapelli plywood seem to have the lowest total moisture content, yet also poplar plywood performs better than other non-durable wood species. Similar conclusions were found by Van den Bulcke et al. (2009b) after 300 days of outdoor ageing of the plywood on the CMM, yet the total moisture accumulation is higher after two years, most probably due to ageing of the plywood. For spruce, maritime pine and poplar (p2 and p3) the distributions are righttailed. For some plywood specimens, a bimodal pattern is observed, most pronounced in coated samples. Reason for this can be found in the division of the exposure period into a warm, dry summer period and a colder and wetter winter period. Furthermore, a phenolic coating reduces the moisture 
201

202

203

204

205

206

207

208

209

210

211

212

213

214

215

216

217

218

219

220

221

222

223

224

225

226

227

228

229

230

231

232

233

234

235

236

237

238

239

240

241

242

243

244

245

246

content of the plywood samples and narrows the distribution. This is particularly obvious for the coated p3 (p3_c), which has a very sharp distribution with nearly constant MC between $13 \%$ and $25 \%$. In order to understand some of the abovementioned phenomena, a subsample was taken from some panels and $\mathrm{X}$-ray scanned. Both 2D slices and 3D renderings are given in Figure 4. All right hand sides of the images as indicated on the first image, were exposed at $45^{\circ}$ degrees and mostly give evidence of severe weathering. Especially on 2D cross-sections the layered structure with glue lines is visible. For the low and high absorbing poplar plywood, there seems hardly any difference in the structure and weathering state when examining the slices. A similar conclusion can be drawn for the birch plywood, yet the cracks in the top veneer of the higher absorbing sample were slightly deeper than for the lower absorbing sample, partially explaining the higher absorbance. For both the lower absorbing p1 and b1 samples, there seems to be a higher amount of glue, brighter and thus denser spots considered being glue, in the top veneer probably blocking water ingress more than is the case for the higher absorbing ones. For spruce plywood the conclusion is evident regarding the cracking of the first glue layer beneath the top veneer for the high absorbing sample. The absence and presence of cracks is shown in Figure 4 by means of white rectangles which give a magnified view (magnification factor is 2.5 ) on these features. Also displayed is the combination of okoumé and poplar showing very distinct glue bands, apparently also contributing to beneficial moisture dynamics, whereas the maritime pine plywood has large cracks in the outer veneer again resulting in a breach of the glue barrier. Bottom images show the sapelli plywood and the almost unaffected coated birch b1 plywood.

The decay risk can be evaluated using the ToW approach. The $20 \%$ and $25 \%$ moisture content limit is used as directional value. Viitanen (1997) suggested that the cumulative sum of periods allowing the decay development (time periods of $\mathrm{RH} 95-100 \%$ and time periods at temperatures above $0-5{ }^{\circ} \mathrm{C}$ ) can be used to predict the risk of decay. According to Rapp et al. (2000) a limit of $25 \%$ wood moisture content seemed to be a reasonable figure beyond which wood can be degraded to a considerable extent, while the lower limit of $20 \%$ MC provides a margin of safety against fungal decay for comparison of the different plywood types (Morris and Winandy 2002). The temperature restriction was set to $5^{\circ} \mathrm{C}$ meaning that days with $\mathrm{T}<5^{\circ} \mathrm{C}$ were not taken into account. Again, as the average of the sample's moisture content is used, it is not possible to pronounce upon the moisture distribution, but in the light of mutual comparison of the samples such an approach is useful. Figure 5 displays the results of ToW analysis for $T$ $>5^{\circ} \mathrm{C}$ and $\mathrm{MC}>25 \%$ (Fig $5 \mathrm{a}$ ) and $\mathrm{MC}>20 \%$ (Fig $5 \mathrm{~b}$ ) after four time periods for the different plywood samples, sorted according to the results after 816 days (June 2006 - August 2008). Clearly the samples with a phenolic film on top stay well below the critical $25 \%$ limit most of the time. Okoumé plywood, even in combination with poplar, belongs to the better performing group, and sapelli is even better than coated plywood species when considering the $20 \%$ MC limit. Spruce, maritime pine and the thicker birch plywood store moisture rather easily, which might increase decay risk. Weathering is an important issue as well, as it is evident that the mutual relations can change of which poplar p3 is a distinct example. Although the latter had the highest absorbance after merely 200 days, this changed drastically after longer ageing, establishing itself at the lower side of the high moisture accumulating specimen. In this respect the long term dynamics are important as the PF glued poplar seems to behave better than the UMF glued type (Fig 5b), whereas this was not evident after one year of outdoor weathering. The same accounts for sapelli, in general even outperforming the coated specimen. The combined poplar plywood with outer okoumé veneer is also remarkable as it seems to have a rather drastic increase in MC > 20\% after more than 800 days, probably caused by failure of the first glue line. All other samples show a rather uniform progression throughout the measuring campaign.

\subsection{Fungal susceptibility}


Fungal test results are presented for two different fungal species and both sealed and non-sealed specimens were tested. For non-sealed specimen in the top graph of Figure 6, high decay levels by Coniophora puteana are noted for the UMF-glued hardwood plywood while the PF-glued hardwood plywood is far more resistant as nearly no mass loss is noted, with b2 as exception. The decay pattern for Pleurotus ostreatus is different. It seems that this fungus is rather glue-indifferent. Decay rates are higher for birch and spruce plywood (>20\%) than for poplar or poplar combination plywood $(<20 \%)$. For the sealed specimen in the bottom graph of Figure 6 , results are clearly different. It is obvious that the fungi have to degrade the substrate starting from the outer veneers without the possibility to enter the layered structure from aside, as the edges are main entrance gates for the non-sealed specimen. The application of an edge sealing alters the situation for Coniophora: overall a positive effect of durable outer veneers or coating application on the plywood durability can be observed, e.g. lower mass loss for the poplar/okoumé combination compared to throughout poplar plywood. By applying an edge sealing the influence of the glue type on decay by Coniophora becomes less explicit. Mass losses are higher with Pleurotus compared to Coniophora when using an edge sealing (Fig. 6). The effect of edge sealing on poplar plywood is small compared to birch plywood. Similar as for Coniophora the protective effect of a coating or the presence of durable outer veneers can be clearly distinguished.

\subsection{Fungal susceptibility and the Brischke model}

Comparing the moisture dynamics of plywood expressed as the dose the specimen received during weathering with the structure of the plywood and the fungal susceptibility of the sealed specimen results in the graph as given in Figure 7 . The $X$-axis of Figure 7 is the accumulated dose received during more than two years of outdoor weathering. Again, these values are used for mutual evaluation as the real distribution throughout the sample is not known. The white dots represent the ratio of the top veneer thickness in regard of the total thickness, which logically increases with increasing moisture dynamics. This is not an unequivocal trend as the b2 plywood deviates from this relationship. Although the latter has a favourable top veneer to total thickness ratio, total dose is higher than expected. Linking total dose to decay of sealed samples by Pleurotus is presented by the black dots. Plywood types receiving high doses do not necessarily have high white rot susceptibility. As stated before, glue type is decisive too concerning both moisture and fungal decay, as can be seen for the p3 plywood. The two groups $A$ and $B$ indicated on the graph, once more illustrate the influence of glue type (PF <-> UMF). Coated specimens are superior to the others. A similar approach for Coniophora was not elaborated due to the amount of missing values.

\section{Discussion}

Performance durability is one of the most important properties of wood-based panels used in housing construction (McNatt and Link 1989; Kajita et al. 1991). It depends on the physico-mechanical functioning as well as the endurance against decay. Both parameters are heavily influenced by ageing and $M C$ of the material. In the present study the $M C$ of 10 different plywood types was measured continuously for more than two years of outdoor weathering. As the X-ray radiographies given in Figure 1 allow assessment of the density distribution of the panel (Chen et al., 2010) and the presence of defects, they can be used as a general inspection tool for the selection of test material. The large knot 
present in one of the spruce panels and the inhomogeneous distribution of densities along the mp panel are the most remarkable features which were not visible at the outside of the panels and can have an influence on the general performance. Crucial is the moisture behaviour of the plywood during ageing and the ageing itself. It is already shown that ageing influences the shear strength (adhesive bonding) of the material (Gillespie and River 1976). Plywood is considered to be substantially a wood-like stable material (Xu and Suchsland 1991). In a study by River (1994), it is shown that plywood retained more than $50 \%$ of initial Modulus of Elasticity (MOE) after 10 years of ageing. Blanchet (2008) showed that plywood scores very well compared to other flooring products such as OSB and HDF when used in environments with changing $\mathrm{T}^{\circ} \mathrm{C}$ and $\mathrm{RH} \%$. Yet, it is to be expected that differences exist between different plywood types and these are indeed very clear when analyzing CMM data. Histogram plotting as shown in Figure 3 already depicts the differences. Softwood species $\mathrm{sp}$ and $\mathrm{mp}$ in general are higher absorbing than hardwood species. For spruce, maritime pine and poplar p2 and p3, with rather thick top veneers, the right tails of the distribution are mostly linked to moisture absorption by this top veneer, but probably not yet with (complete) failure of the glue lines. The samples indicated as high absorbing within their group, especially the spruce sample, clearly have a more severe failure of the glue line beneath the top veneer, as shown by X-ray tomography scanning in Figure 4. Weathering of the rather thick top veneer causes checking and stress at the glue line and manifests itself through cracks. For spruce the stress difference between early- and latewood might have caused an extra force inducing rupture of the glue plane. For the higher absorbing birch sample, cracking of the top veneer probably was the starting point for higher absorption in combination with failure of the glue surface between this top veneer and the underlying layer, causing water to penetrate and to accumulate. For poplar, a similar conclusion can be drawn from visual and $X$-ray inspection of the top veneer, showing cracks and erosion spots. X-ray tomography is a very valuable tool for inspection of the failure modes and quality of the plywood before and during weathering. On volumes scanned at higher resolution it would even be possible to map the glue distribution in order to use it as a quantifiable criterion for durability. The application of a coating reduces the moisture content in general, yet failure of the coating might lead to moisture accumulation beneath the coating in the veneers, making the subsequent drying of the material difficult. The beginning of such an effect can be seen on the coated p1_c with a shift towards a higher moisture content frequency. As stated before, the measured $\mathrm{MC}$ is an average of the whole panel and as such it is not possible to derive a distribution of the moisture throughout the sample. Therefore electrical resistance sensors (Brischke et al., 2008) should be inserted beneath each veneer layer to closely monitor the moisture profile in order to be able to characterize breaching of the glue lines or coating layer.

Within the framework of decay risk, the ToW concept is used here to assess and compare the different plywood types. When expounding this in the light of progressing ageing of the samples, one gets a clear view on failure of veneer or glue. A low and a high absorbing group can be distinguished in Figure 5a and $5 \mathrm{~b}$, which is especially for the $20 \%$ graph already established after 204 days of weathering. In fact these groups can be considered to be the moisture dynamics of nearly intact specimen. Mutual relations are not yet fixed and during weathering changes occur progressively. The mixed okoumé and poplar plywood panel is an example of pronounced changing moisture dynamics. After more than 600 days of weathering, it shifted to the lower absorbing group although it started in the higher absorbing one. This evolution underpins the importance of long-term outdoor weathering, which might be completed with artificial weathering tests.

Another important aspect of the performance durability concept is the decay susceptibility of a material. Chung et al. (1999) gave evidence that resistance of plywood mainly depends on the anatomical characteristics of the wood species used in the wood veneer with a preference of brown and white rot 
respectively for soft- and hardwood. The latter result was not found in this study, but anatomical characteristics or wood species definitely play a role. The question can be raised whether or not the ENV 12038 set up is resulting in an over-severe assessment of plywood durability, since the presence of a coating or durable outer veneer induces no difference in the test results obtained. Therefore the application of an edge sealing to all test specimens should be considered. A proper edge sealing of plywood in test material is vital since edges of panel products are more susceptible to water uptake than their faces, due to the end grain exposure of timber veneers (Lea and Berry 1995). In service situations, most plywood products are used as whole boards with a large surface area, whereas test specimens are rather small and have a high ratio of edge to surface area. The use of small sealed test specimens could lead to a more realistic test approach, particularly when test specimen are coated or have a durable top veneer. In fact, an outer veneer such as okoumé can be considered to behave as a coating. The decay results support the argument to include edge sealing requirements in the ENV 12038 test methodology. Plywood used outdoors is often coated and edge sealing is recommended. These precautions deliver a higher resistance to degradation which is not materialized in results obtained according to the current standard test methodology. Zanuttini et al. (2003) also concluded that the best protection for plywood is a surface treatment and coating on the edges and therefore the ENV 12038 set up is not representing a realistic outdoor situation. Furthermore, from these results it can also be concluded that while performing an ENV 12038 durability assessment, at least one brown rot fungus and one white rot fungus, and even different strains (Kernergang and Grinda, 1984) should be included in the test set up. Protection of plywood against decay might include preservative treatment, yet Van Acker and Stevens (1989) have shown that this kind of treatment does not give sufficient protection against white and brown rot fungi. Tascioglu and Tsunoda (2010) have subjected azole-treated plywood to fungal attack and found better results for hardwood plywood but not for plywood made from softwood. It is expected that these treatments do not change the moisture dynamics of the wood, but induce an inhibitory effect towards fungi. Moisture accumulation reduction is of course the first goal to strive for and with a correct choice of veneer thickness, glue and wood species one already reaches a considerable level of protection.

\section{Conclusions}

Within the framework of low-energy and low-cost construction, the use of plywood as one of the major re-engineered wood products is an important market. When used in use class 3 situations, both physicomechanical performance as well as biological durability are important. There are clear differences in moisture dynamics and fungal susceptibility between different plywood types. Linking fungal laboratory tests and outdoor weathering with continuous moisture measurements is not straightforward, but they can better be considered as complementary methods. It is clear that intrinsic durability testing (ENV 12038) of the used wood species is not sufficient for the assessment of plywood. Even low durable wood species perform better than expected and moisture dynamics can contribute significantly to this understanding through concepts such as time of wetness and dose-response. Furthermore, X-ray tomography as an inspection tool is absolutely invaluable to detect defects and failure modes. Further investigation on the fungal susceptibility and its relation with the moisture dynamics and ageing of the plywood necessitates the implementation of a system as worked out by Brischke and Rapp (2008) and Brischke et al. (2008) with covered plywood to follow the phenomena in moist conditions while measuring material temperature and following degradation evolution. 


\section{Acknowledgements}

383 This research was performed in support of the European Research Project "Biological performance 384 testing methodology to evaluate the durability of plywood as a quality indicator for exterior construction 385 purposes", with acronym Plybiotest (QLK5-CT-2002-1270). The authors would like to thank Mr. Rik De 386 Rycke for his technical assistance. The authors also wish to thank the Fund for Scientific Research387 Flanders (Belgium) for the postdoctoral funding granted to the first author. 
Blanchet, P., 2008. Long-term performance of engineered wood flooring when exposed to temperature and humidity cycling. Forest Products Journal 58, 37-44.

Brischke, C., Rapp, A.O., 2008. Dose-response relationships between wood moisture content, wood temperature and fungal decay determined for 23 European field test sites. Wood Science and Technology 42, 507-518.

Brischke, C., Rapp, A.O., Bayerbach, R., 2008. Measurement system for long-term recording of wood moisture content with internal conductively glued electrodes. Building and Environment 43, 1566-1574.

Chen, S.G., Liu, X.H., Fang, L.M., Wellwood, R., Digital X-ray analysis of density distribution characteristics of wood-based panels. Wood Science and Technology 44, 85-93.

401

Chung, W.Y., Wi, S.G., Bae, H.J., Park, B.D., 1999. Microscopic observation of wood-based composites exposed to fungal deterioration. Journal of Wood Science 45, 64-68.

De Smet, J., Van Acker, J., 2006. Testing methodology and CE-marking for biological performance of plywood for exterior construction purposes. COST E44 and E49. Wood Resources and Panel Properties, Valencia, Spain.

Dinwoodie, J.M., 2000. Timber: Its nature and behaviour. E \& FN Spon, London.

EN 335-3. 1995. Durability of wood and wood-based products. Definition of hazard classes of biological attack - Part 3: Application to wood-based panels. European Committee for Standardisation (CEN), Brussels, Belgium.

EN 636. 2003. Plywood - Specifications. European Committee for Standardisation (CEN), Brussels, Belgium.

412 ENV 1099. 1998. Plywood - Biological durability. Guidance for assessment of plywood for use in the 413 different hazard classes. European Committee for Standardisation (CEN), Brussels, Belgium.

414 ENV 12038. 1999. Durability of wood and wood-based products - Wood-based panels - Method for 415 determining resistance against wood-destroying basidiomycetes. European Committee for 416 Standardisation (CEN), Brussels, Belgium.

417 FAO, 2009. State of the world's forests. FAO, Rome, Italy.

418 FEIC 2009. Annual Report 2008-2009 presented at the General Assembly in Santiago de Compostella, 419 Spain.

420 Gillespie, R.H., River, B.H., 1976. Durability of adhesives in plywood. Forest Products Journal 26, 21-25.

421 Kajita, H., Mukudai, J., Yano, H., 1991. Durability evaluation of particleboards by accelerated ageing tests. 422 Wood Science and Technology 25, 239-249. 
423 Kernergang, W., Grinda, M., 1984. Testing the resistance of plywood against wood destroying 424 Basidiomycetes. Holz Als Roh-Und Werkstoff 42, 41-49.

425 Krüger, E.L., Laroca, C., 2010. Thermal performance evaluation of a low-cost housing prototype made 426 with plywood panels in Southern Brazil. Applied Energy 87, 661-672.

427 Lea, R., Berry, R., 1995. Evaluating the resistance of wood-based panel products to fungal attack. IRG/WP 428 95-20071. The International Research Group on Wood Preservation, Stockholm, Sweden.

429 McNatt, J.D., Link, C.L., 1989. Analysis of ASTM D-1037 accelerated-aging test. Forest Products Journal $43039,51-57$.

431 Morris, P.I., Winandy, J.E., 2002. Limiting conditions for decay in wood systems. IRG/WP 02-10421. The 432 International Research Group on Wood Preservation, Cardiff, South Wales, UK.

433 Rapp, A.O., Peek, R.D., Sailer, M., 2000. Modelling the moisture induced risk of decay for treated and 434 untreated wood above ground. Holzforschung 54, 111-118.

435 River, B.H., 1994. Outdoor Aging of Wood-Based Panels and Correlation with Laboratory Aging. Forest 436 Products Journal 44, 55-65.

437 Tascioglu, C., Tsunoda, K., 2010. Biological performance of copper azole-treated wood and wood-based 438 composites. Holzforschung 64, 399-406.

439 Van Acker, J., De Smet, J., 2007. Moisture dynamics of plywood in exterior applications as a basis for 440 service life prediction. Proceedings of the International Panel Products Symposium, Cardiff, Wales.

441 Van Acker, J., Stevens, M., 1989. Treatment of poplar plywood with solvent and waterborne 442 preservatives. IRG/WP/3538. The International Research Group on Wood Preservation, Stockholm, 443 Sweden.

444 Van den Bulcke, J., Boone, M., Van Acker, J., Stevens, M., Van Hoorebeke, L., 2009a. X-ray tomography as 445 a tool for detailed anatomical analysis. Annals of forest science 66, 508.

446 Van den Bulcke, J., Van Acker, J., De Smet, J., 2009b. An experimental set-up for real-time continuous 447 moisture measurements of plywood exposed to outdoor climate. Building and Environment 44, 2368$448 \quad 2377$.

449 Viitanen, H.A., 1997. Modelling the time factor in the development of brown rot decay in pine and 450 spruce sapwood - The effect of critical humidity and temperature conditions. Holzforschung 51, 99-106.

$451 \mathrm{Xu}, \mathrm{H} .$, Suchsland, O., 1991. The expansion potential - a new evaluator of the expansion behavior of 452 wood composites. Forest Products Journal 41, 39-42.

453 Zanuttini, R., Nicolotti, G., Cremonini, C., 2003. Poplar plywood resistance to wood decay agents: efficacy 454 of some protective treatments in the light of the standard ENV 12038. Annals of Forest Science 60, 8345589. 
458 Table 1

459 Plywood types used with details on wood species, glue type, veneer composition and number of replicates 460 per veneer type

\begin{tabular}{|c|c|c|c|c|c|c|}
\hline Code & Wood species & Glue $^{a}$ & \# Plies & Thickness [mm] & Veneers $^{b}$ & \# Replicates \\
\hline p1 & poplar & UMF & 7 & 15 & $1.3 / 2.6 / 2.6$ & 5 \\
\hline $\mathrm{p} 1 \mathrm{c}^{*}$ & poplar & UMF & 7 & 15 & $1.3 / 2.6 / 2.6$ & 1 \\
\hline p2 & poplar & UMF & 9 & 18 & $2.1 / 2.1 / 2.1$ & 3 \\
\hline p3 & poplar & PF & 9 & 18 & $2.1 / 2.1 / 2.1$ & 3 \\
\hline p3_c* & poplar & PF & 9 & 18 & $2.1 / 2.1 / 2.1$ & 1 \\
\hline b1 & birch & PF & 11 & 15 & $1.4 / 1.4 / 1.4$ & 6 \\
\hline b1_c* & birch & PF & 11 & 15 & $1.4 / 1.4 / 1.4$ & 1 \\
\hline b2 & birch & PF & 10 & 20 & $1.1 / 2.3 / 2.3$ & 1 \\
\hline $\mathrm{sp}$ & spruce & PF & 5 & 15 & $3.0 / 3.0 / 3.0$ & 6 \\
\hline ok1 & okoumé & UMF & 7 & 15 & $1.0 / 3.0 / 2.0$ & 3 \\
\hline ok2 & okoumé + poplar & PF & 7 & 15 & $1.0 / 3.0 / 2.0$ & 1 \\
\hline $\mathrm{mp}$ & maritime pine & PF & 7 & 17 & $2.5 / 2.5 / 2.5$ & 3 \\
\hline sap & sapelli & PF & 9 & 15 & $1.0 / 3.0 / 2.0$ & 3 \\
\hline
\end{tabular}

461

$462{ }^{a}$ PF: Phenol Formaldehyde glue / UMF: Ureum fortified Melamine Formaldehyde glue

$463 \quad{ }^{b}$ Veneer thickness [mm]: top veneer / inner cross / inner core

$464 *$ plywood coated with a phenolic film 
467 Table 2

468 Recorded weather data for the period June 2006 - August 2008

\begin{tabular}{lcccc}
\hline & Mean & St. Dev. & Range & Cumulative \\
\hline Rain [mm] & 0.1 & 0.6 & $0 \rightarrow 32.6$ & 1844.8 \\
RH [\%] & 71.1 & 18.3 & $16.2->100$ & \\
Temperature $\left[{ }^{\circ} \mathrm{C}\right]$ & 13.6 & 6.7 & $-4.4 \rightarrow 38.2$ & \\
Wind speed $[\mathrm{km} / \mathrm{h}]$ & 12.1 & 18.3 & $0 \rightarrow 135.9$ & \\
Solar intensity $\left[\mathrm{W} / \mathrm{m}^{2}\right]$ & 114.0 & 199.1 & $0->1108.8$ & $7.3\left[\mathrm{GJ} / \mathrm{m}^{2}\right]$ \\
\hline
\end{tabular}

469

470 
471 Figure 1. X-ray radiographies of the plywood types. The two circular features on every board are the bore 472 holes of the screws used for attachment of the boards on the T-shaped holder.

473 Figure 2. Climate data and MC (\%) of $\mathrm{mp}$.

474 Figure 3. Histograms of the MC (\%) of the plywood types and indication of the $20 \%$ and $25 \%$ MC limits. 475 Samples with different moisture dynamics within the same plywood species are subdivided in a low and 476 high absorbing group with separate histograms.

477 Figure 4. X-ray tomography scans and accompanying cross-section of some selected plywood types. 478 Samples with different moisture dynamics within the same plywood species are subdivided in a low and 479 high absorbing group and from each group a representative sample is scanned. Rectangles give a 480 magnified view $(2 x)$ on the first glue line, with apparent glue line cracking in the higher absorbing 481 samples. Scale bar $=5 \mathrm{~mm}$.

482 Figure 5. Time of Wetness (ToW) concept: the Y-axis represents the number of days a certain plywood 483 species has an MC above $25 \%$ (a) or $20 \%$ (b). The ToW is calculated for 4 different periods: 0 -> 204 days, $4840->408$ days, 0 -> 612 days, 0 -> 816 days.

485 Figure 6. Mass loss (\%) of sealed and non-sealed plywood. ${ }^{*}=$ missing values. Black $=$ Coniophora 486 puteana, grey $=$ Pleurotus ostreatus .

487 Figure 7. Accumulated dose in relation to the ratio of top veneer thickness and total plywood thickness 488 (right Y-axis) and mass loss (\%) induced by Pleurotus ostreatus of sealed plywood samples. Black circles = 489 mass loss, white circles = ratio top veneer / total thickness. 

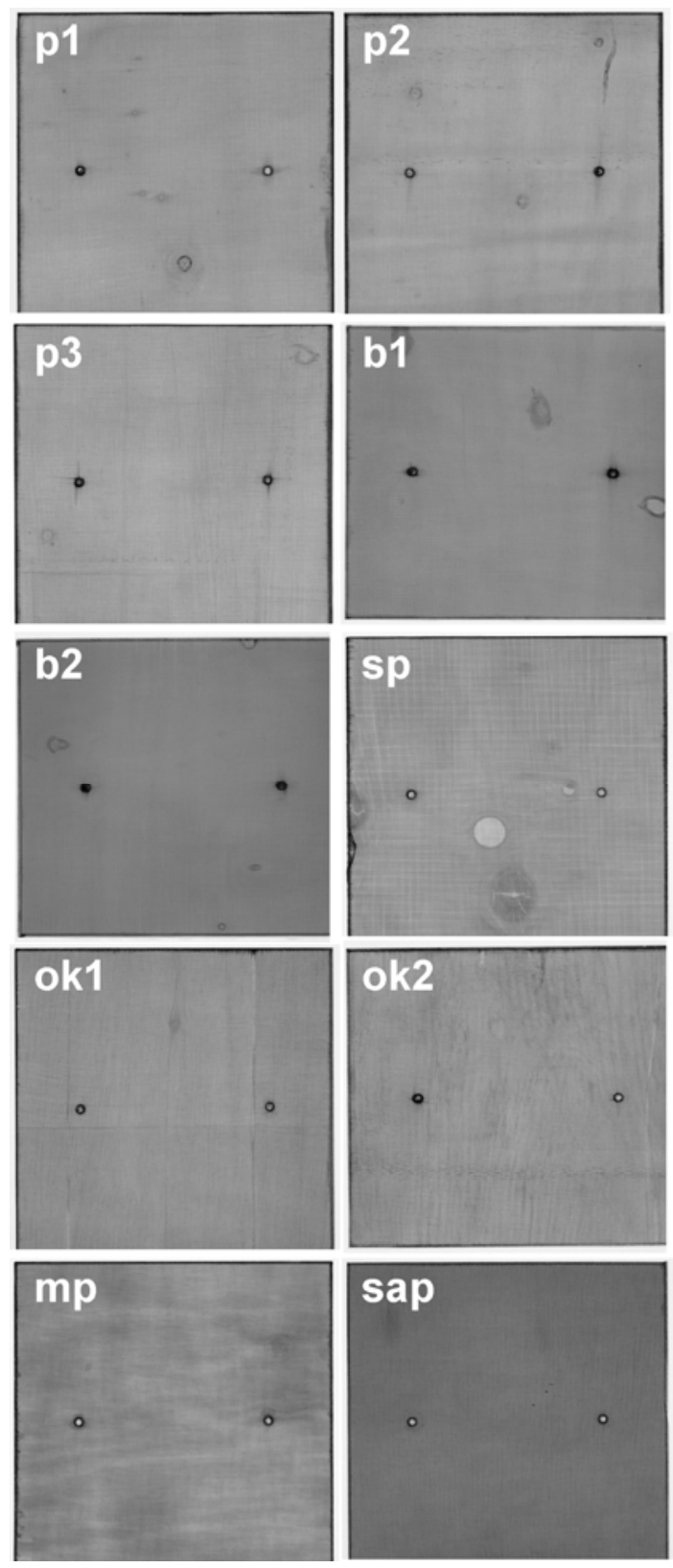

491

Figure 1

493 

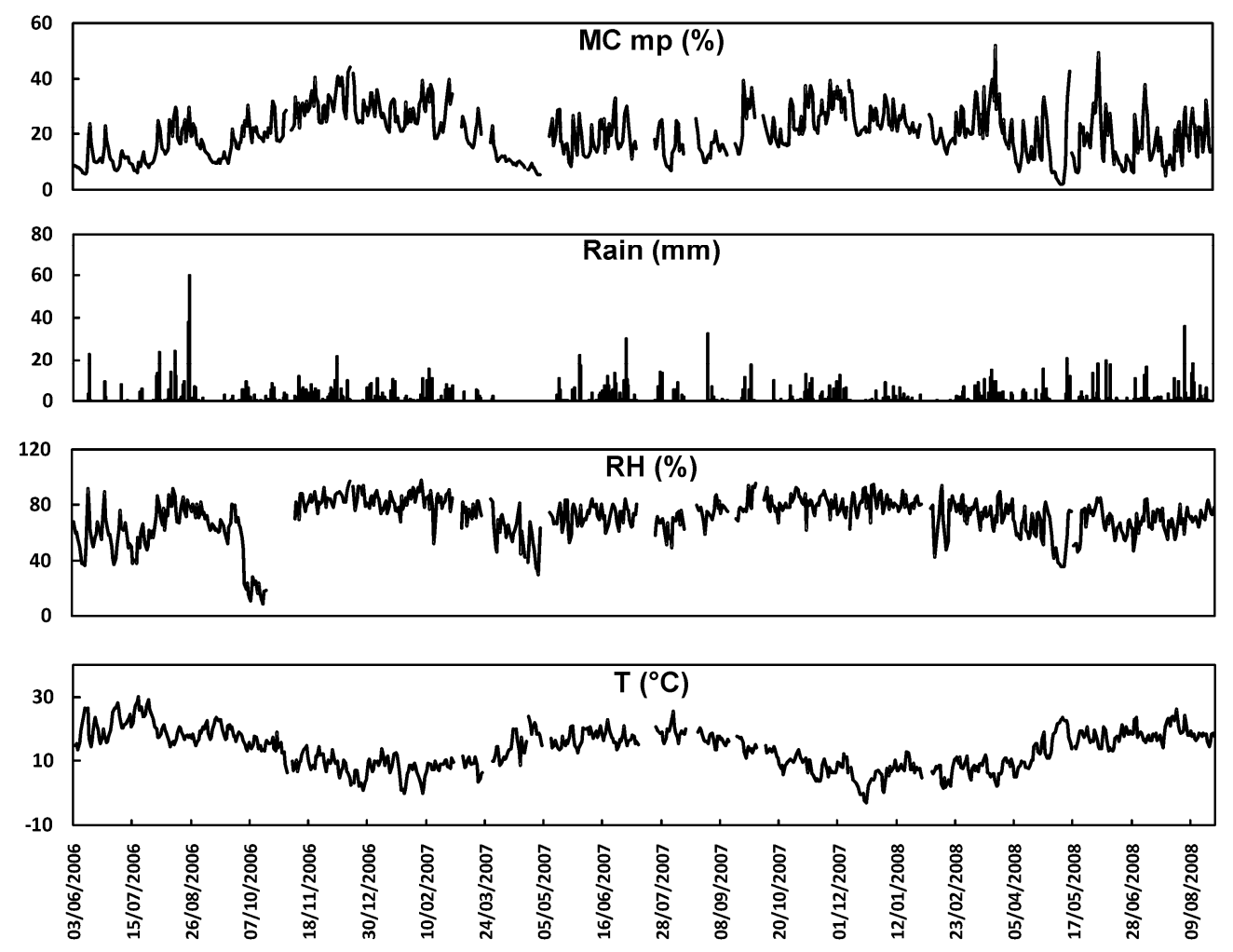

$495 \quad$ Figure 2 

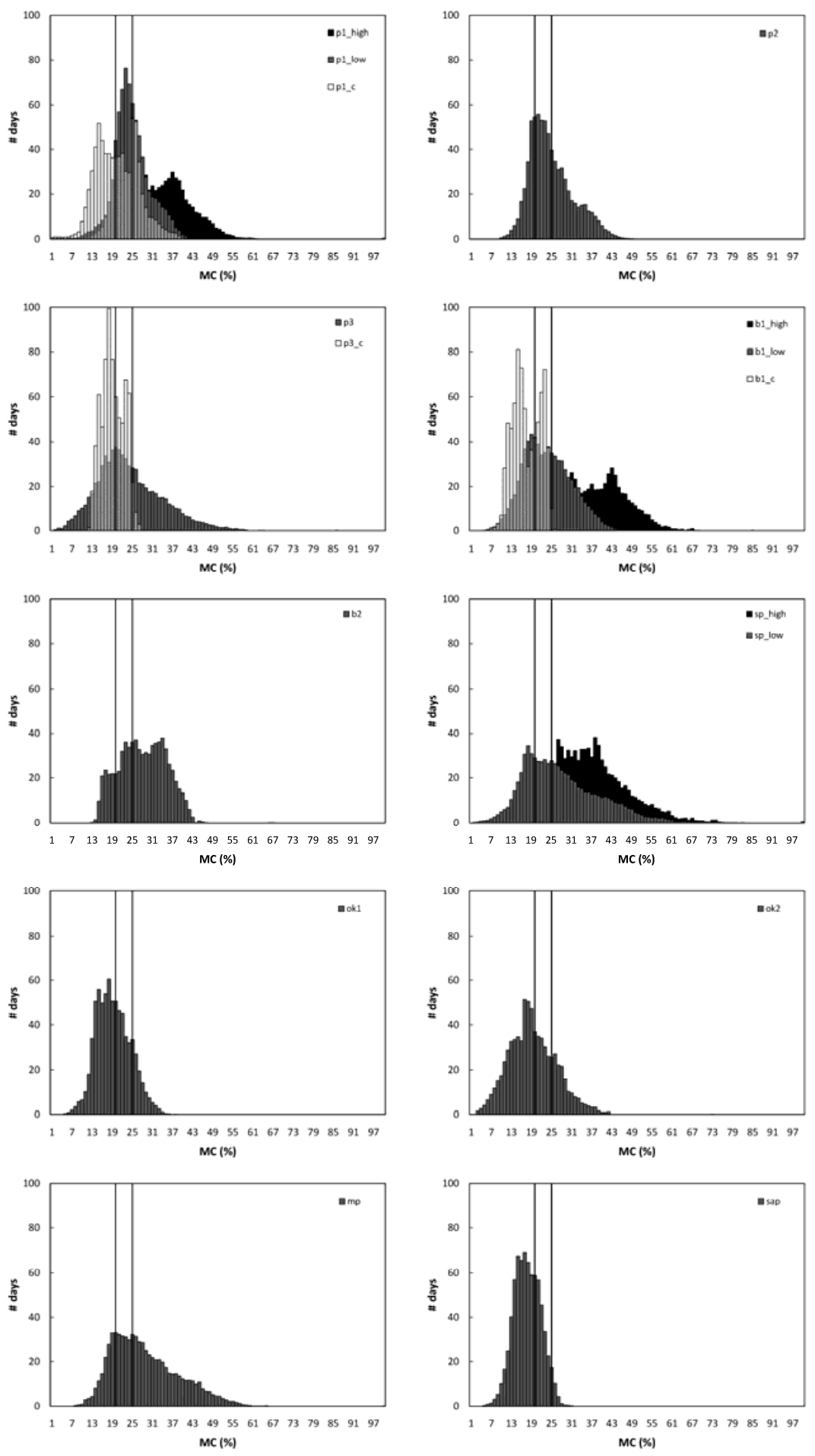

Figure 3 

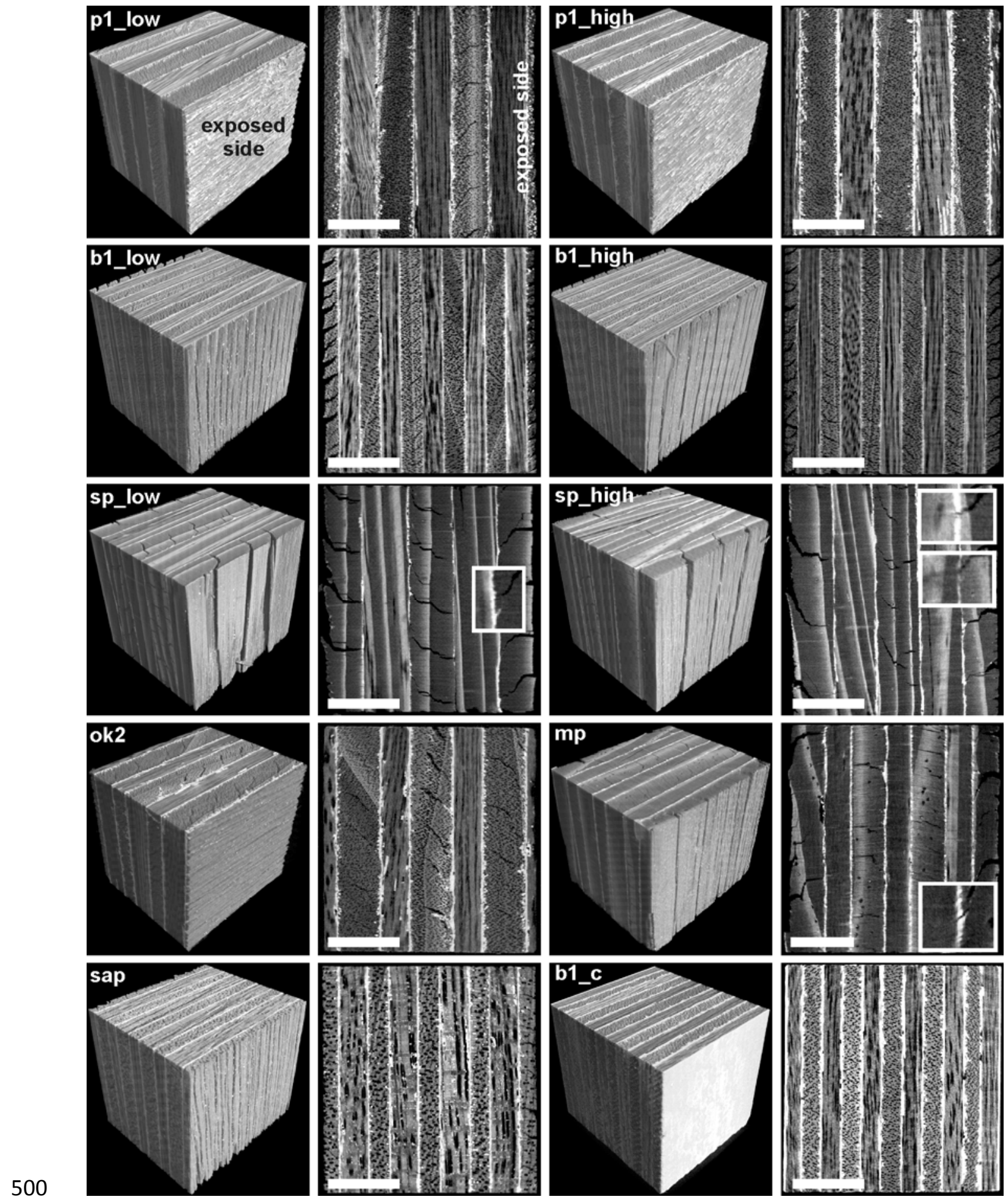

501

Figure 4

502 


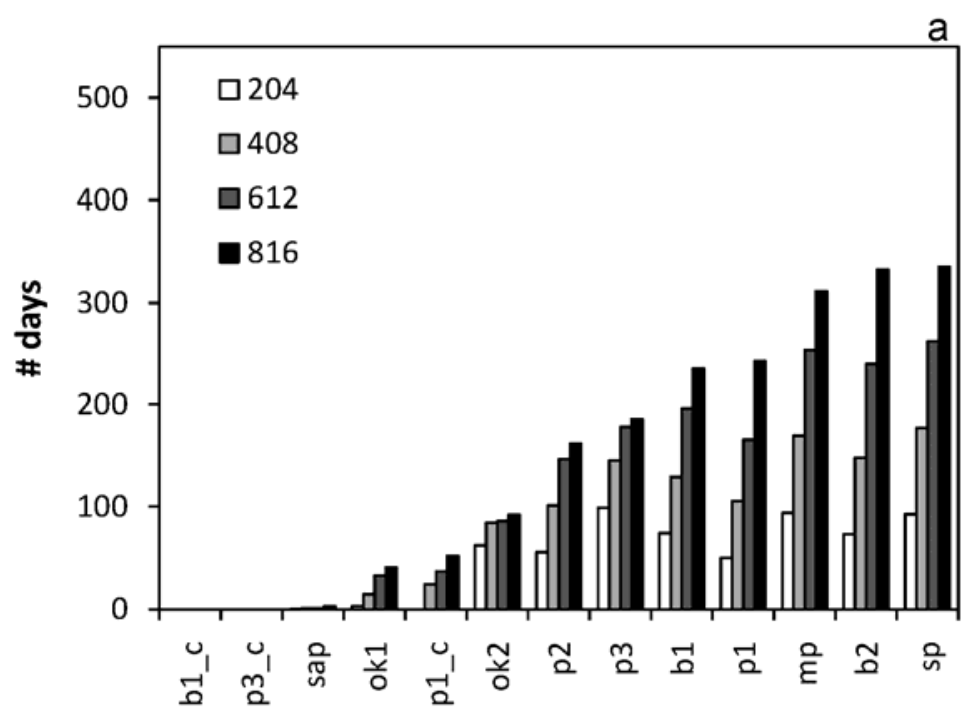

Plywood

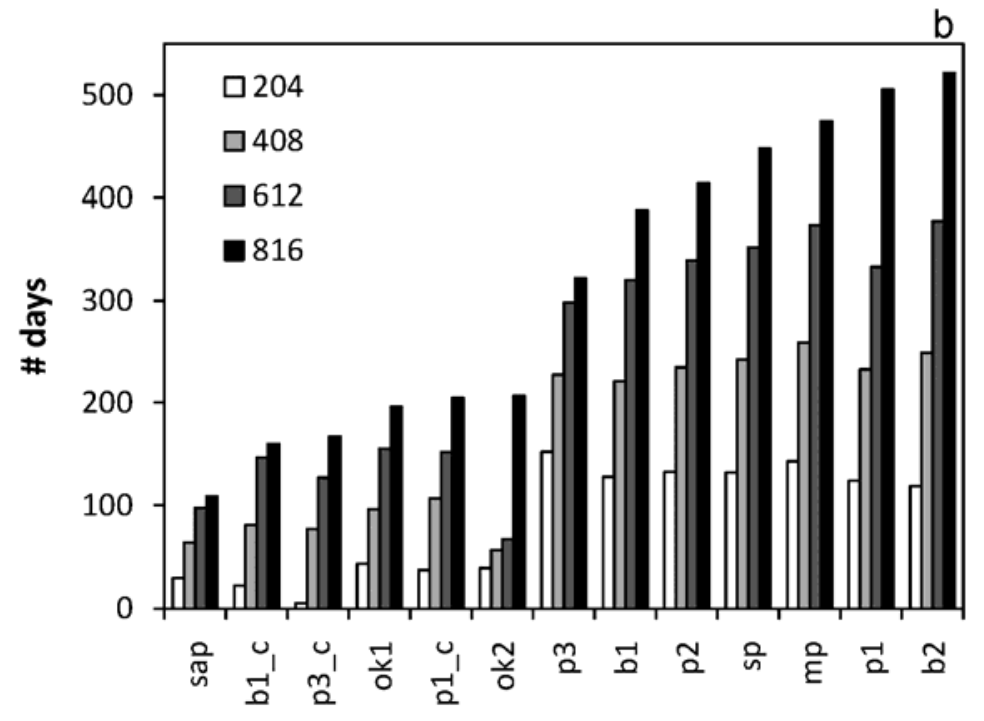

503

Plywood

504 Figure 5

505 

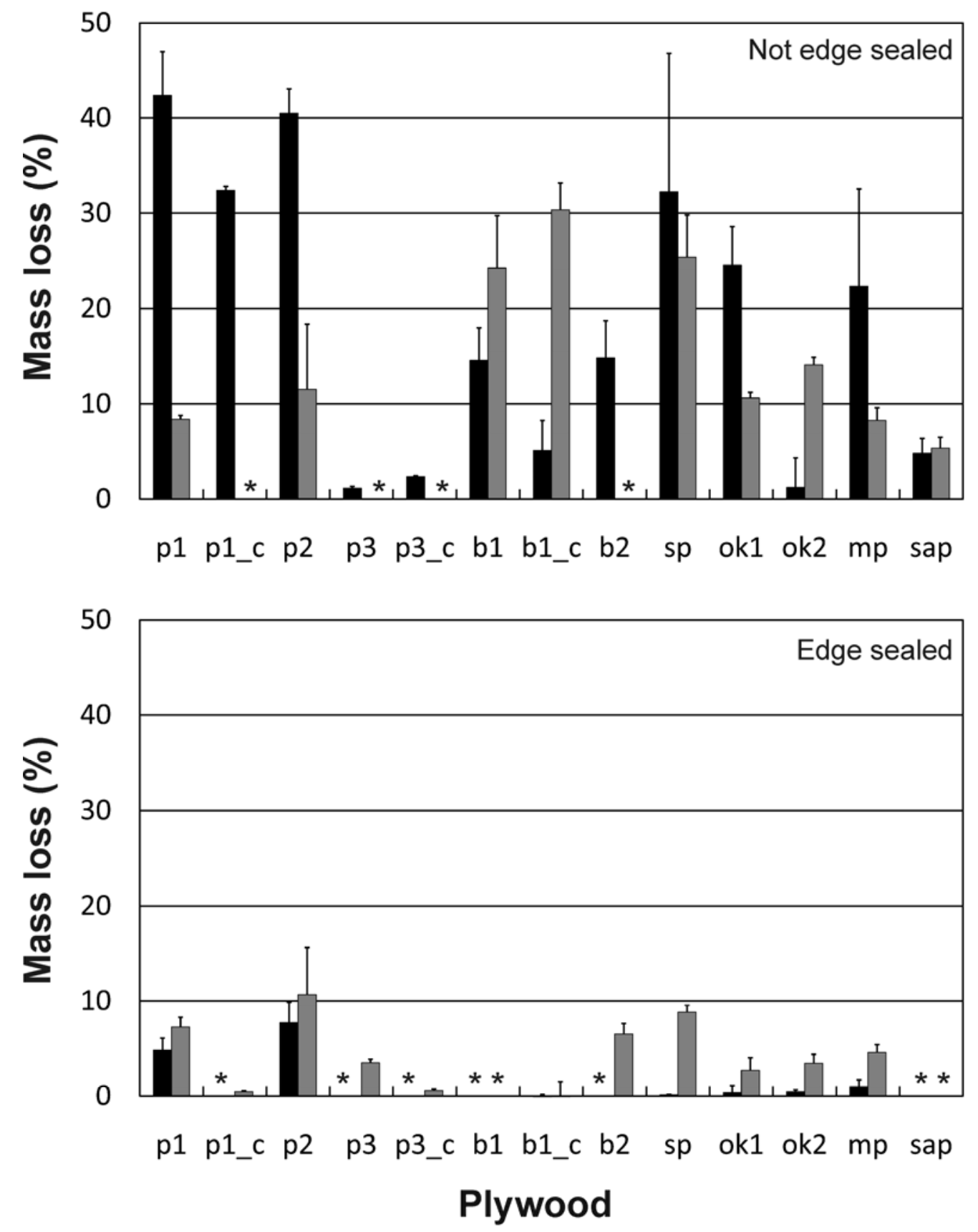

Figure 6

508 


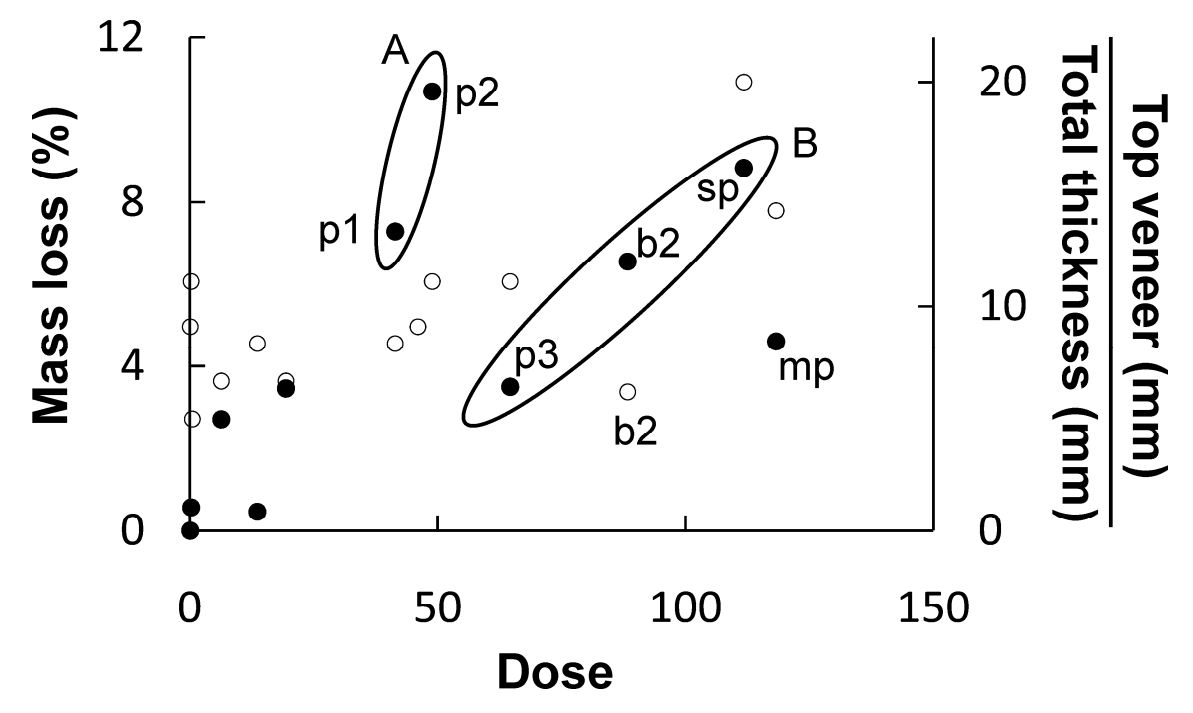

$510 \quad$ Figure 7

511 